\title{
VARIACIÓN DE LA DIVERSIDAD DE AVES DE SOTOBOSQUE EN EL PARQUE NACIONAL LAGOS DE MONTEBELLO, CHIAPAS, MÉXICO
}

\author{
José Luís Rangel-Salazar1, Paula L. EnríQuez y Eugenia C. Sántiz LóPez \\ Departamento de Ecología y Sistemática Terrestre, El Colegio de la Frontera Sur (ECOSUR), \\ Apartado Postal 63- 29290, San Cristóbal de Las Casas, Chiapas, MÉXICO. \\ ${ }^{1}$ Autor para correspondencia: jlrangel@ecosur.mx
}

Rangel-Salazar, J. L., P. L. Enríquez y E. C. Sántiz-López. 2009. Variación de la diversidad de aves de sotobosque en el Parque Nacional Lagos de Montebello, Chiapas, México. Acta Zoológica Mexicana (n. s.), 25(3): 479-495

RESUMEN. Para entender la variación espacial y temporal de la diversidad de aves de sotobosque, evaluamos la diversidad de especies en cuatro sitios (Las Grutas, Yalmutz, Vivero y Yalhuech) con diferentes tipos de vegetación y manejo dentro del Parque Nacional Lagos de Montebello, Chiapas, de julio de 1997 a junio de1998. Identificamos 71 especies de aves: 50 fueron residentes y 21 migratorias. Las familias mejor representadas fueron Parulidae (15), Tyrannidae (9) y Turdidae (8). La mayor proporción de especies registradas fueron exclusivas para un solo sitio (63.4\%) y tan solo 9.8\% se distribuyó en los cuatro sitios. Las mayores frecuencias relativas de captura total fueron para Chlorospingus ophtalmicus (0.197), Catharus ustulatus (0.110), Lampornis viridipallens (0.083) y Myioborus miniatus (0.072). El número de capturas varió entre los cuatro sitios estudiados, donde el bosque mixto de coníferas y latifoliadas (Vivero) presentó los valores máximos y el bosque mesófilo (Las Grutas) los mínimos. Yalmutz tuvo la mayor riqueza de especies (36), y Las Grutas la menor (24). La mayor diversidad derivada del reciproco de Simpson (16.41) y uniformidad (0.68), se registraron en Las Grutas, que fue el sitio menos perturbado y con los últimos remanentes de bosque mesófilo de montaña. Sin embargo, Yalmutz presentó los valores más altos en diversidad de Shannon (4.37), así como el mayor número tanto de especies exclusivas (14) como raras (21). Yalmutz presentó una mezcla de elementos de coníferas y latifoliadas, y fue el segundo sitio con un menor grado de perturbación por actividades antropogénicas. La mayor riqueza de especies y abundancia de individuos se presentó durante la temporada seca. El Parque Nacional Lagos de Montebello presenta una importante diversidad de especies de aves por la variación en ambientes y grados de perturbación que presenta. Sin embargo, la persistencia de las poblaciones de aves y su conservación en la región dependerá tanto de la permanencia de ambientes no perturbados y de la calidad de los ambientes secundarios y manejados.

Palabras clave: aves de sotobosque, riqueza de especies, comunidades biológicas, bosques mesófilos, bosques de pino-encino.

Rangel-Salazar, J. L., P. L. Enríquez, and E. C. Sántiz-López. 2009. Diversity variation in understory forest birds in Lagos de Montebello National Park, Chiapas, Mexico. Acta Zoológica Mexicana (n. s.), 25(3): 479-495

Recibido: 04/06/2008; aceptado: 08/09/2009. 


\begin{abstract}
To understand the spatial and temporal variation of the understory bird diversity, we estimated species diversity in four sites (Las Grutas, Vivero, Yalmutz y Yalhuech) with different vegetation type and management regimes in the Lagos de Montebello National Park, Chiapas from July 1997 to June 1998. We identified 71 bird species; 50 residents and 21 migrants. Families with more species were Parulidae (15), Tyrannidae (9) and Turdidae (8). Most of species were recorded to a single site $(63.4 \%)$ and only $9.8 \%$ were distributed in all four sites. The highest relative frequencies of total captures were for Chlorospingus ophtalmicus (0.197), Catharus ustulatus (0.110), Lampornis viridipallens (0.083) and Myioborus miniatus (0.072). Capture number varied among sites, while Vivero showed the maximum values, Las Grutas showed the minimum. Yalmutz had the highest species richness (36), and Las Grutas the lowest (24). The highest diversity (Simpson reciprocal 16.41) and evenness (0.68) was recorded in Las Grutas, which was the site less disturbed and showed one of the last remnants of montane cloud forest. However, Yalmutz showed the highest values of Shannon diversity (4.37), and high values of both exclusive (14) and rare species (21). Yalmutz showed a mix of elements of pines and broadleaves and it was the second site with less disturbance by human activities. The higher species richness and abundance was during the dry season. The National Park Lagos de Montebello showed an important bird species diversity for the environmental variation and different stage of disturbance. The persistence of bird populations and their conservation in the region will depend on the remaining of nondisturbed environments and the quality of secondary and managed forests.
\end{abstract}

Key words: Understory birds, species richness, biological communities, cloud forests, pine-oak forests.

\title{
INTRODUCCIÓN
}

La diversidad biológica se distribuye de manera heterogénea en el mundo. Esta distribución es una de las características mas importantes que definen a las comunidades biológicas. Existen factores geográficos, evolutivos, históricos y ecológicos que determinan esta heterogeneidad (Rickelfs \& Schluter 1993). Sin embargo, procesos como la pérdida, degradación y fragmentación de los ambientes naturales modifican los patrones de distribución, abundancia y composición de especies de las comunidades en prácticamente todos los ambientes. La reducción y transformación de los ambientes naturales ocasionan la simplificación de la estructura y composición de sus comunidades biológicas.

Algunas especies de aves están restringidas a un determinado tipo de vegetación, y esto establecerá su habilidad para persistir en ambientes transformados (Johns 1991). Las especies que podrían estar más afectadas son aquellas del interior de bosque que por lo regular no pueden cruzar áreas abiertas (Johns 1991). La persistencia de las especies dependerá de la disponibilidad de hábitat y en ocasiones más de un hábitat puede ser requerido (Fahrig y Nuttle 2005). El estado de Chiapas puede considerarse como una de las áreas más ricas en especies de aves a nivel nacional (Escalante-Pliego et al. 1998), en donde se han descrito alrededor de 659 especies de aves (Rangel-Salazar et al. 2005). Sin embargo, pocos o nulos estudios se han realizado sobre diversidad de aves y su variación espacial y temporal en las Áreas Naturales Protegidas como el Parque Nacional Lagos de Montebello.

El Parque Nacional Lagos de Montebello presenta una superficie de 6022 ha (Carlson 1954), y hasta hace unas décadas contaba con una importante cobertura 
vegetal compuesta principalmente por bosques mesófilo de montaña y de coníferas (Flores-Villela \& Geréz 1994). Sin embargo, para 1993 se había perdido más del $60 \%$ de la cobertura vegetal como consecuencia del disturbio natural y antropogénico (March \& Flamenco 1996). Aunado a esto, los incendios en 1998 provocaron graves daños dentro del parque, al afectar alrededor de 800 hectáreas de bosque de pinoencino- liquidambar en buen estado (SEMARNAP 1998). A pesar de esta alarmante alteración y pérdida de áreas boscosas de la cobertura vegetal, poca información se ha generado sobre la diversidad de fauna que existe en el parque (e.g. Horvart et al. 2001). El propósito de este estudio fue evaluar la composición y variación espaciotemporal de la diversidad de aves de sotobosque en cuatro sitios con diferente tipo de vegetación y manejo, para entender las respuestas de la diversidad de aves a los efectos de los procesos de alteración ambiental en la diversidad biológica, de tal manera de considerar estos efectos en las estrategias de conservación.

\section{MATERIAL Y MÉTODOS}

El estudio se realizó en el Parque Nacional Lagos de Montebello que se encuentra en la Altiplanicie del estado de Chiapas, México (16 $06^{\prime} 40^{\prime \prime}$ y $16^{\circ} 10^{\prime} 20^{\prime \prime} \mathrm{N}$; $91^{\circ}$ 37'40" y 91 47'47" O; Figura 1). La altitud del parque oscila entre los 1500 y 1 $800 \mathrm{msnm}$. El clima es semi-cálido con lluvias en verano según la clasificación de Köppen modificado por García (1973). La precipitación es de 300 a $400 \mathrm{~mm}$ en la época de secas (noviembre-abril) y entre 1200 a $1400 \mathrm{~mm}$ durante la época de lluvias (mayo a octubre) (INEGI 1984). La temperatura promedio anual es de $18^{\circ} \mathrm{C}$. La vegetación del Parque es un mosaico de asociaciones vegetales donde sobresale el bosque de pino-encino liquidámbar, bosque de pino, bosque mesófilo de montaña, vegetación secundaria y riparia (Rzedowski 1978).

De julio de 1997 a junio de 1998 se realizaron capturas de aves en cuatro sitios en el parque que difirieron en tipo de vegetación y estructura: 1) Las Grutas presentó elementos de bosque mesófilo de montaña, la más alta diversidad vegetal, mayor altitud y pendiente en relación a los otros tres sitios. Este sitio presentó especies arbóreas como Malmea sp, Parathesis bellizensis y Exothea paniculata, y un estrato arbustivo conformado principalmente por individuos juveniles de las especies Chamaedorae sp, Psychotria marginata y Podocarpus matudai (Zarco 2000). 2) Yalmutz presentó una mezcla de elementos de coníferas con especies de latifoliadas. En este sitio se encontraron principalmente especies de árboles como Quercus sapotaefolia, Myrica cerifera y Clusia sp. y arbustos de Miconia mexicana, Lyonia squamulosa y Pallicourea galleottiana. 3) Vivero presentó también mezcla de coníferas y latifoliadas. Aunque difirió de Yalmutz por presentar menor cobertura arbórea y diversidad vegetal, conformado principalmente por Quercus laurina, Clethra suaveolens y Parathesis bellizensis, y en el estrato arbustivo Calliandra houstoniana, Pallicourea pasiflora y Miconia mexicana. 4) Yalhuech presentó 
bosque de pino-encino, menor altitud y pendiente que el resto de los sitios. El estrato arbóreo está dominado por especies como C. suaveolens, Pinus oocarpa y $Q$. sapotaefolia, y el estrato arbustivo por Eupatorium semialatum, Verbesina apleura y P. pasiflora (Cuadro 1; Zarco 2000).

Nuestra predicción fue que la riqueza y diversidad de especies de aves, que involucra la distribución de las abundancias de individuos dentro de las especies (Pueyo 2006), se asociará negativamente a un gradiente de perturbación que incide en la composición de especies y en la estructura de la vegetación (Ricklefs 1977). La

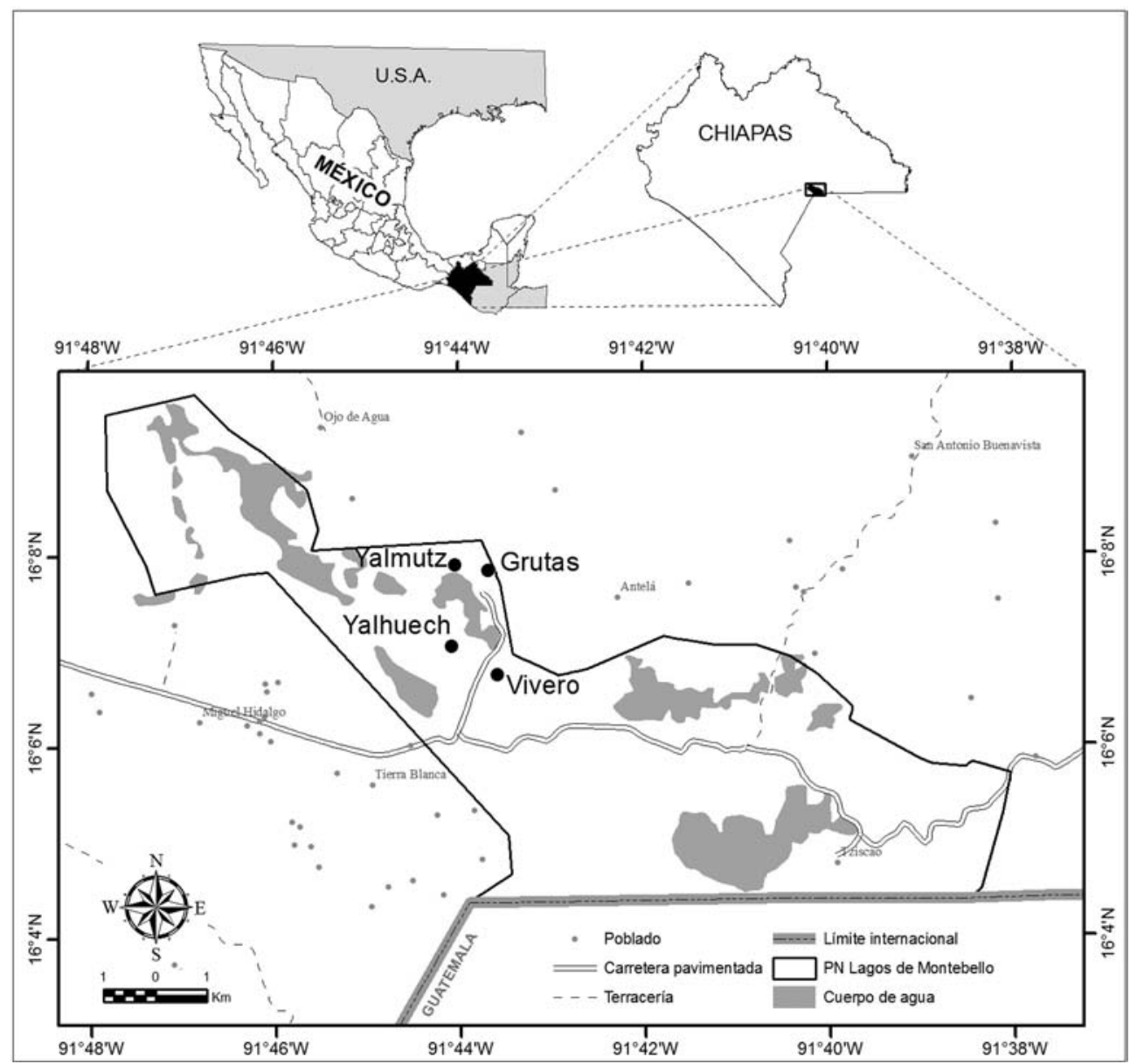

Figura 1. Localización geográfica de los cuatro sitios estudiados (Las Grutas, Vivero, Yalmutz y Yalhuech) dentro del Parque Nacional Lagos de Montebello, Chiapas, México. 
estructura como la altura de los árboles y la cobertura arbórea y arbustiva son variables que claramente difieren entre los sitios de muestreo (Zarco 2000). El grado de perturbación debido a actividades antropogénicas varió entre los sitios estudiados. Las Grutas fue el sitio menos perturbado, con un valor de perturbación acumulable de uno (Cuadro 2), mientras que Yalhuech fue el sitio con mayor perturbación con respecto al resto (valor 15), ya que existieron evidencias de intensas actividades como extracción de madera, fuego, ganadería y cacería (Cuadro 2). Yalmutz presentó grados de perturbación moderada con poca extracción de madera y cacería moderada (valor 5), mientras que en Vivero la extracción de madera fue más intensa y presentó poca ganadería (valor 10; Zarco 2000).

Cuadro 1. Características ambientales de los cuatro sitios de muestreo en el Parque Nacional Lagos de Montebello, Chiapas.

\begin{tabular}{lcclcc}
\hline Sitios & $\begin{array}{c}\text { Pendiente } \\
\left({ }^{\circ}\right)\end{array}$ & $\begin{array}{c}\text { Altitud } \\
(\mathbf{m s n m})\end{array}$ & Vegetación & $\begin{array}{c}\text { Abundancia } \\
\text { florística* }\end{array}$ & $\begin{array}{c}\text { Diversidad } \\
\text { florística** }\end{array}$ \\
\hline Las Grutas & 15 & 1465 & Bosque mesófilo & 892 & 6.63 \\
Yalmutz & 12 & 1460 & Coníferas-liquidambar & 1469 & 5.74 \\
Vivero & 9 & 1445 & Coníferas-liquidambar & 2850 & 3.97 \\
Yalhuech & 8 & 1280 & Pino-encino & 212 & 5.49 \\
\hline
\end{tabular}

* Individuos de flora/cuadrante; **Inverso índice dominancia Simpson (Zarco 2000).

Cuadro 2. Valores cualitativos de la perturbación de la vegetación en cuatro sitios en el Parque Nacional Lagos de Montebello, Chiapas.

\begin{tabular}{lcccc}
\hline & \multicolumn{4}{c}{ S I T I O S } \\
\hline Variables & Las Grutas & Yalmutz & Vivero & Yalhuech \\
\hline \multirow{2}{*}{ Fuego } & 0 & 0 & 0 & 3 \\
Ocoteo & 0 & 2 & 3 & 3 \\
Extracción Madera & 0 & 1 & 3 & 3 \\
Ganadería & 0 & 0 & 1 & 3 \\
Cacería & 1 & 2 & 3 & 3 \\
Valor Total & 1 & 5 & 10 & 15 \\
\hline
\end{tabular}

Criterios: Ausente (0), Ligero (1), Moderado (2), Intenso (3) 
En cada sitio de muestreo se ubicaron parcelas de 90x90m, donde se colocaron 9 redes de niebla, repartidas en 3 líneas rectas con 3 redes cada línea. Las líneas de redes estuvieron separadas por $45 \mathrm{~m}$. Las redes se abrieron antes del amanecer hasta el medio día $(n=45$ horas/red/día), durante tres días consecutivos por sitio (135 horas/red/mes/sitio). Cada sitio fue muestreado cada 2 meses durante un año. Las aves capturadas se identificaron con guías de campo (e.g., National Geographic 1983, Pyle et al. 1987, Howell \& Webb 1995). El patrón de movimiento anual (status), y la clasificación de los diferentes gremios tróficos, se realizó de acuerdo a Terborgh et al. (1990), para determinar la composición de especies residentes y migratorias de la comunidad de aves por sitio y su ubicación trófica. La variación anual de las aves migratorias es un proceso crucial en la comunidades de aves en la región tropical y puede ser diferencial a diferentes escalas espaciales.

Las características de la comunidad de aves para el total y por sitio se expresaron como la riqueza acumulada de especies capturadas, el número de especies exclusivas a un solo sitio, las especies raras en las que se capturó un individuo por sitio, y la abundancia relativa representada por la frecuencia de captura de cada especie entre el total de capturas (Karr 1985). La diversidad, equitatividad y uniformidad se estimó con el inverso de Simpson, el índice de Shannon y el índice de uniformidad de Simpson's (Brower et al. 1977, Krebs 2001). También se estimó el índice de Morisita-Horn para evaluar la similitud entre sitios en función de la composición y abundancia de las especies. Los valores de este índice oscilan entre 0 (no hay similitud) y 1 (máxima similitud) (Krebs 1989).

Para determinar la variación espacio-tiempo la riqueza y abundancia de las especies se separaron en sitios y épocas: secas (noviembre-abril) y lluvias (mayooctubre). El análisis de varianza paramétrica se utilizó para determinar la variación de la riqueza de especies y la diversidad entre los sitios y las temporadas. Previo a los análisis, las variables cumplieron con los supuestos estadísticos de distribución normal y similitud de varianzas con las pruebas de Shapiro-Wilks (W) y Bartlett, respectivamente (Gotelli y Ellison 2004). Todos los análisis estadísticos se realizaron con el programa JMP en SAS 5.1 (Sall et al. 2005). Las pruebas se consideraron significativas cuando a $\leq 0.05$.

\section{RESULTADOS}

Un total de 518 capturas en 71 especies fueron registradas en el Parque Nacional Lagos de Montebello, Chiapas, México (en un esfuerzo total de captura de 3110 horas/red). Del total de especies capturadas, 50 especies (70.4\%) fueron residentes y 21 (29.6\%) migratorias Neotropicales (Cuadro 3). Las familias que presentaron un mayor número de especies fueron Parulidae (15), Tyrannidae (9) y Turdidae (8).

Del total de especies registradas, 45 (63.4\%) fueron exclusivas a un solo sitio, y $26(36.6 \%)$ se presentaron en más de un sitio. Solamente siete especies $(9.9 \%)$ se 
distribuyeron en los cuatro sitios, y de las cuales cuatro fueron las que presentaron las más altas frecuencias relativas de captura en todo el estudio: Chlorospingus ophtalmicus (0.197), Catharus ustulatus (0.110), Lampornis viridipallens (0.083), y Myioborus miniatus (0.072; Cuadro 3). Especies migratorias Neotropicales capturadas con elevadas frecuencias relativas de captura fueron Wilsonia pusilla (0.066) y Seiurus aurocapilla (0.033; Cuadro 3).

La media de individuos capturados varió entre los cuatro sitios estudiados, donde Vivero (bosque de coníferas y latifoliadas) presentó los valores mayores, y Las Grutas (bosque mesófilo) los menores $\left(F_{3,23}=4.24, P<0.05\right.$; Cuadro 4). Con respecto a la riqueza de especies, Yalmutz (bosque de coníferas y latifoliadas) presentó la mayor riqueza con 36, y Las Grutas la menor con 24. Sin embargo, no existieron diferencias en la distribución del número de especies entre los cuatro sitios estudiados $\left(F_{3,23}=2.29, P=0.10\right.$; Cuadro 3$)$. Aunque las curvas de acumulación de especies durante el periodo de estudio mostraron un incremento en los cuatro sitios, ninguna se estabilizó al terminar el muestreo (Figura 2).

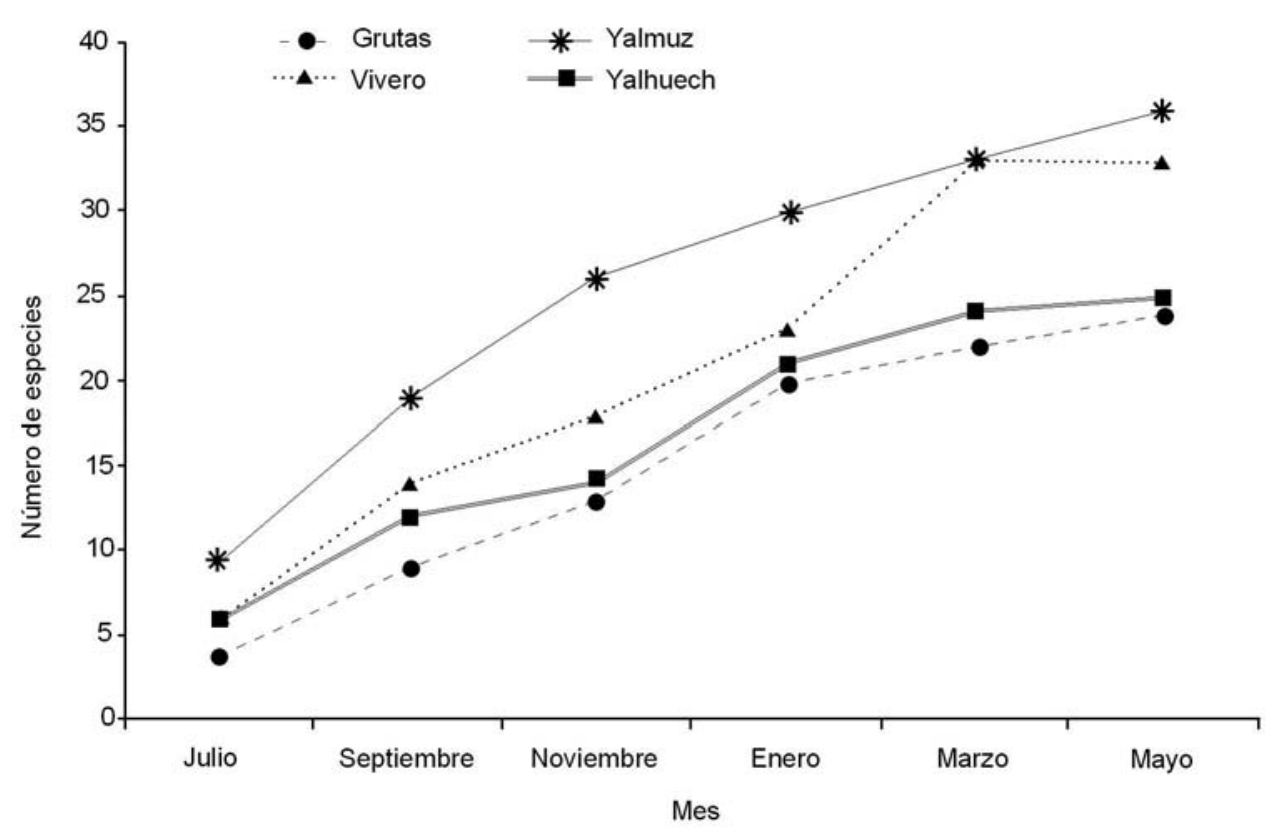

Figura 2. Curvas de acumulación de especies en cuatro sitios (Las Grutas, Vivero, Yalmutz y Yalhuech) en el Parque Nacional Lagos de Montebello, Chiapas. De julio de 1997 a junio de 1998. 
Rangel-Salazar et al.: Aves de sotobosque del Parque Nacional Lagos de Montebello

Cuadro 3. Número de individuos de especies de aves capturadas en cuatro sitios del Parque Nacional Lagos de Montebello, Chiapas, México (Julio 1997 - Junio 1998). Status (Patrones de Movimiento Anual): $\mathrm{R}=$ Especie Residente, $\mathrm{M}=$ Especie migratoria (migratoria Neotropical). Gremio Trófico:

$\mathrm{RD}=$ Rapaz diurna, $\mathrm{RN}=$ Rapaz Nocturna, GT= Granívoro Terrestre, FT= Frugívoro Terrestre, $\mathrm{NA}=$ Nectarívoro, OA= Omnívoro Arbóreo, FA= Frugívoro Arbóreo, IAS= Insectívoro Arbóreo Buscador, ITG= Insectívoro Terrestre Recogedor, IBS= Insectívoro Buscador de Corteza, ITS= Insectívoro Buscador Terrestre, IAG= Insectívoro Arbóreo Recogedor. Frecuencia de captura (individuos capturados por especie/ total de individuos de todas las especies).

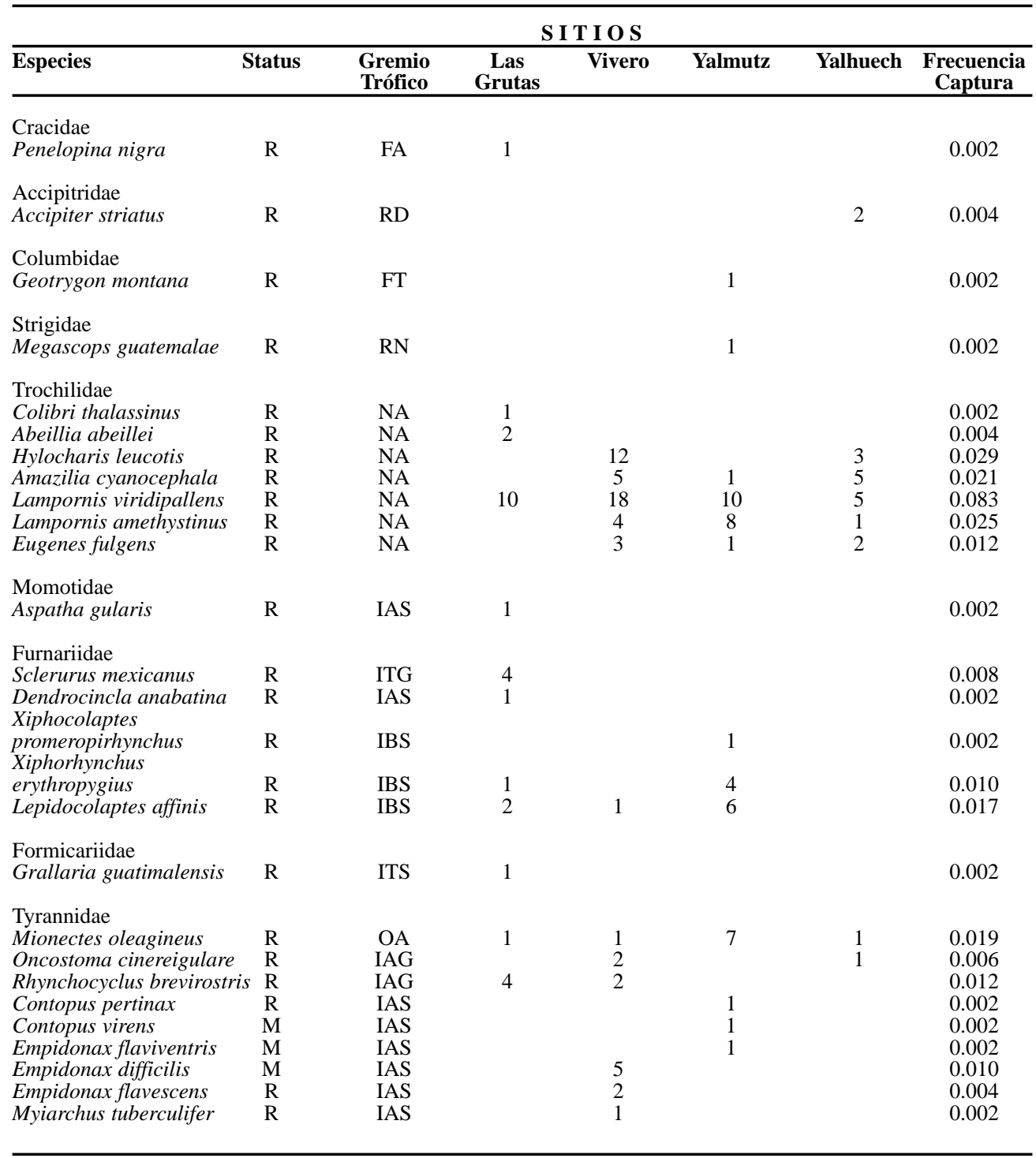


Vireonidae

Vireo solitarius (solitarius) R

Vireo philadelphicus $\quad \mathrm{M}$

Cyclarhis gujanensis $\quad \mathrm{R}$

IAG

IAG

IAG

Corvidae

Cyanolyca cucullata

Cyanolyca pumilo

$\mathrm{R}$
$\mathrm{R}$

OA

OA

Troglodytidae

Campylorhynchus zonatus

Thryothorus maculipectus

Troglodytes aedon

Henicorhina leucophrys

1

0.004

0.002

0.004

Turdidae

Myadestes occidentalis

Myadestes unicolor

Catharus aurantiirostris

Catharus mexicanus

Catharus dryas

Catharus ustulatus

Hylocichla mustelina

Turdus grayi

$\mathrm{R}$
$\mathrm{R}$
$\mathrm{R}$
$\mathrm{R}$
$\mathrm{R}$
$\mathrm{M}$
$\mathrm{M}$
$\mathrm{R}$

FA

FA

FT

FT

FT

OA

FT

OA

IAG

Dumetella carolinensis

$\mathrm{M}$

Melanotis hypoleucus

$\mathrm{R}$

IAG

0.002

0.002

Parulidae

Vermivora chrysoptera

Parula superciliosa

Dendroica virens

Mniotilta varia

Protonotaria citrea

Helmitheros vermivorus

Seiurus aurocapilla

Seiurus noveboracensis

Oporornis formosus

Oporornis tolmiei

Wilsonia pusilla

Wilsonia canadensis

Myioborus miniatus

Basileuterus culicivorus

Basileuterus rufifrons

M

$\mathrm{R}$

$\mathrm{M}$

$\mathrm{M}$

$\mathrm{M}$

$\mathrm{M}$

$\mathrm{M}$

M

M

M

M

$\mathrm{M}$

$\mathrm{R}$

$\mathrm{R}$

$\mathrm{R}$

IAG

IAG

IAG

IAG

IAG

ITG

ITG

ITG

IAG

IAG

IAG

IAG

IAG

FA

FA

FA

1
2
1
4
2
2
1

5

1

0.002

0.008

0.002

1

0.012

Thraupidae

Chlorospingus ophthalmicus $\mathrm{R}$

ITS

ITS

GT

GT

6

17

6

Emberizidae

Atlapetes albinucha $\quad \mathrm{R}$

Buarremon brunneinucha

Melozone biarcuata

$\mathrm{R}$

Melospiza lincolnii

$\mathrm{R}$

FA

GT

GT

2

1

1

Cardinalidae

Pheucticus ludovicianus

Passerina cyanea

$\mathrm{M}$
$\mathrm{M}$

Passerina ciris

M

$\mathrm{R}$

$\mathrm{OA}$

1

0.002

0.008

Fringillidae

Icteridae 
La mayor diversidad (reciproco de Simpson; 16.41) y uniformidad (0.68), se registraron en Las Grutas. Sin embargo, Yalmutz presentó los valores más altos en diversidad de Shannon (4.37), así como mayor número tanto de especies exclusivas (14) como raras (21; Cuadro 4). De acuerdo a la similitud entre sitios, ambos bosques de coníferas y latifoliadas (Vivero y Yalmutz) presentaron los mayores porcentajes (93\%), seguido de Vivero y Yalhuech (bosque de pino-encino) (82\%), estos sitios compartieron 16 especies, respectivamente. Los sitios con menor similitud, fueron Las Grutas (bosque mesófilo) con Yalhuech (28\%), al compartir solo 7 especies (Cuadro 5). Estos últimos sitos fueron los que contrastaron en los valores cualitativos de perturbación, donde el bosque mesófilo presentó los menores valores (1) y el bosque de pino-encino los mayores (15; Cuadro 2).

Cuadro 4. Valores de diversidad y equitatividad en cuatro sitios en el Parque Nacional Lagos de Montebello, Chiapas, México (Julio 1997 - Junio 1998). H/R= Horas/Red,

$\mathrm{S}=$ Riqueza total de especies, Número total de individuos capturados, $\mathrm{N}_{2}=$ Recíproco de Simpson,

$\mathrm{H}_{1}=$ Índice de Shannon-Weiner (base 2), Uniformidad de Simpson, Sp.= Especies exclusivas, $\mathrm{R}=$ especies raras (número de especies con un individuo capturado) por sitio.

\begin{tabular}{lcccccccc}
\hline Sitio & H/R & $\mathbf{S}$ & Individuos & $\mathbf{N}_{\mathbf{2}}$ & $\begin{array}{c}\text { Shannon } \\
\mathbf{H}_{\mathbf{1}}\end{array}$ & Uniformidad & $\mathbf{S p .}$ & $\mathbf{R}$ \\
\hline Las Grutas & 810 & 24 & 53 & 16.41 & 4.13 & 0.68 & 11 & 13 \\
Yalmutz & 790 & 36 & 127 & 14.60 & 4.37 & 0.41 & 15 & 21 \\
Vivero & 765 & 33 & 208 & 9.96 & 3.96 & 0.30 & 12 & 10 \\
Yalhuech & 745 & 25 & 130 & 9.42 & 3.76 & 0.38 & 7 & 9 \\
Total & 3110 & 71 & 518 & 13.67 & 4.67 & 0.19 & 45 & 53 \\
\hline
\end{tabular}

El bosque mesófilo fue el sitio menos perturbado y presentó 11 especies exclusivas, las cuales son residentes y especialistas a este tipo de bosque, 7 se consideraron raras por presentar un solo individuo capturado (Cuadro 3). El bosque de coníferas y latifoliadas (Yalmutz) presentó 15 especies exclusivas, de las cuales 14 tuvieron un solo individuo capturado y de estas 5 fueron migratorias Neotropicales. El otro bosque de coníferas y latifoliadas (Vivero) presentó 12 especies exclusivas, de las cuales 6 fueron raras y de estas 4 migratorias Neotropicales. Yalhuech que fue el sitio más perturbado de los cuatro, presentó 7 especies exclusivas, de las cuales 5 fueron raras y de estas tres fueron especies migratorias Neotropicales. El bosque mesófilo presentó solo 2 especies migratorias Neotropicales, mientras que el resto de los sitios Yalmutz, Vivero y Yalhuech presentaron 12, 13 y 10 especies migratorias respectivamente. 
Cuadro 5. Valores del índice de similitud de Morisita-Horn (número de especies compartidas), en cuatro sitios del Parque Nacional Lagos de Montebello, Chiapas.

\begin{tabular}{lcccc}
\hline & Las Grutas & Yalmutz & Vivero & Yalhuech \\
\hline Las Grutas & 1 & $0.47(12)$ & $0.38(10)$ & $0.28(7)$ \\
Yalmutz & & 1 & $0.93(16)$ & $0.73(14)$ \\
Vivero & & 1 & $0.82(16)$ \\
Yalhuech & & & 1 \\
\hline
\end{tabular}

En los análisis temporales, la época seca (o periodo de aves migratorias neotropicales) presentó tanto el mayor número de individuos (secas $=345$ vs. lluvias $=173 ; F_{1,23}=6.92, P<0.05$ ), como de especies (secas $=51$ vs. lluvias $=43 ; F_{1}$, ${ }_{23}=12.27, P<0.01$ ). Respecto a los gremios tróficos, el mayor número de especies fueron insectívoras con el $53.5 \%$, particularmente los insectívoros arbóreos. Las especies frugívoras fueron el segundo gremio trófico más abundante $(15.5 \%$ del total; Cuadro 3). Los frugívoros arbóreos representaron el mayor número de individuos (25.9\%), seguido de los insectívoros arbóreos recogedores (29.3\%), y de los nectarívoros $(17.6 \%)$.

\section{DISCUSIÓN}

Las especies de aves de sotobosque registradas en los cuatro sitios en el Parque Nacional Lagos de Montebello, representaron el 19.4\% del total reportadas para Chiapas (659 especies; Rangel-Salazar et al. 2005). La mayoría de las especies fueron residentes y cerca del $30 \%$ fueron migratorias latitudinales. El área de Montebello es una de las regiones de Mesoamérica donde se concentran un importante número de especies de aves migratorias latitudinales (Rappole \& Tipton 1992).

Dentro de la comunidad de aves de sotobosque, algunas especies presentaron una mayor representatividad en cuanto al número de individuos capturados y mayor distribución. Chlorospingus ophtalmicus estuvo bien representada, y se ha reportado que realiza migraciones altitudinales (Winker et al. 1997). Esta especie ha sido registrada como abundante en algunos bosques mesófilos de Chiapas como la Reserva de la Biosfera El Triunfo y en Guatemala (Gómez de Silva et al. 1999, Eisermann \& Schulz 2005). Catharus ustulatus es una especie migratoria Neotropical y también presentó un gran número de capturas. Esta especie se ha considerado abundante durante la migración tanto en regiones bajas del Caribe, como en tierras altas, como El Triunfo (Gómez de Silva et al. 1999). Lampornis viridipallens y Myoborus miniatus también presentaron una amplia distribución en 
los sitios estudiados. Esta última especie ha sido considerada común en sus áreas de distribución de bosques húmedos de pino-encino y encino (Stiles \& Skutch1989, Howell \& Webb1995).

De los cuatro sitios estudiados dentro del parque, el bosque de coníferas y latifoliadas (Yalmutz) presentó la mayor riqueza de especies, el mayor número tanto de especies raras (especies con un individuo), como migratorias Neotropicales. Además, fue el segundo sitio que presentó una mayor diversidad (Shannon) y uniformidad. Este sitio presenta una mezcla de elementos de coníferas y latifoliadas, y mayor cobertura arbórea que Vivero, además fue el segundo sitio con un menor grado de perturbación por actividades antropogénicas (i.e. poca extracción de madera, ocoteo; el cual consiste en cortar pequeños pedazos de madera rica en resina, que se utilizan como combustible para prender fogatas y fogones, y cacería moderada; Cuadro 2).

Por otra parte, el bosque mesófilo (Las Grutas) presentó los valores mayores de diversidad de especies (reciproco de Simpson) y de homogeneidad de los cuatro sitios, pero la menor riqueza de especies. Las Grutas tuvo la mayor diversidad vegetal con respecto a los otros sitios (Cuadro 1; Zarco 2000). Fue el segundo sitio con elevados valores de diversidad de Shannon, así como el segundo con especies raras, y con solo dos especies migratorias Neotropicales, las cuales son poco comunes en este tipo de bosque (Gómez de Silva et al. 1999). Las Grutas representa uno de los últimos remanentes de bosque mesófilo de montaña dentro del Parque Nacional Lagos de Montebello, este sitio presentó los niveles más bajos de perturbación, además que no ha sido severamente afectado por los incendios forestales (Zarco 2000).

El bosque de coníferas y latifoliadas (Vivero), presentó el mayor número de individuos capturados y fue el segundo sitio con mayor riqueza de especies. Este bosque aunque presentó la menor diversidad vegetal, tuvo la cobertura arbórea más alta (Cuadro 1). El bosque de pino-encino (Yalhuech) presentó el menor número de especies de aves raras, menos especies exclusivas, menor diversidad de aves y homogeneidad que los demás sitios. Yalhuech fue el sitio más perturbado y degradado que el resto, ha sufrido quemas e incendios con anterioridad, así como pastoreo. A pesar de esto, dos especies migratorias Neotropicales (C. ustulatus y W. pusilla) fueron las más abundantes en este sitio. La primera de ellas es una especie que utiliza este sitio de paso durante la migración, y la segunda permanece en el sitio durante todo el período migratorio.

La diversidad total registrada (reciproco de Simpson) en el Parque Nacional Lagos de Montebello fue de 13.67, donde el bosque mesófilo presentó la diversidad máxima (16.41). Otros estudios en áreas Neotropicales, como en la Selva Lacandona en Chiapas, México se ha reportado una diversidad de 20.66 para aves de sotobosque (Rangel-Salazar et al. 1993). Aunque los tipos de vegetación son diferentes en 
composición florística (Rzedowski 1978), así como en condiciones topográficas y climáticas, la alta diversidad de las comunidades de aves tropicales se ha atribuido parcialmente a la estructura de la vegetación como determinante en la estructura y organización (Terborgh 1985). Sin embargo, factores antropogénicos también están determinando la composición y diversidad en estos ambientes (Johns1991).

Las especies con una mayor distribución geográfica tienden a ser las más abundantes localmente que aquellas especies con distribución restringida (Hanski et al. 1993). De las 71 especies registradas, siete especies presentaron una amplia distribución en los sitios de muestreo. Cuatro de estas especies fueron las más abundantes en todo el estudio. Las especies con distribución restringida representaron el $63 \%$ del total de especies. Esta relación de distribución y abundancia tiene importantes implicaciones para el estudio y conservación de la diversidad de aves en la región. Sobre todo, porque las acciones de conservación deben de ser soportadas en procesos de priorización de aquellas especies con una distribución restringida y que son poco abundantes.

En los bosques tropicales, la mayoría de las especies de aves son raras (Karr 1990). En este estudio el 44\% del total de especies (31) fueron raras. En algunos casos, esta rareza se puede presentar por la distribución en parches de las especies, porque estas pueden estar ampliamente distribuidas pero son raras a través de su rango de distribución, o porque son especialistas o restringidas a un determinado ambiente (Karr 1990). El bosque de coníferas y latifoliadas (Yalmutz) presentó el mayor número de especies raras (21 especies), seguido de los otros sitios con alrededor de la mitad \pm 10 . Sin embargo, la proporción de especies raras a través de distintas áreas tropicales varía sustancialmente. Por ejemplo, en la Selva Lacandona, Chiapas, se reportó que el $47 \%$ de las especies fueron raras (Rangel-Salazar et al. 1993), de la misma forma en un bosque tropical lluvioso en Perú, Terborgh et al. (1990) registraron el $42 \%$ de las especies como raras. Karr (1977) en un bosque tropical lluvioso de Panamá, registró el 31\% como raras. Karr et al. (1990) en bosques tropicales lluviosos citaron el 19\% de especies raras para Costa Rica, 20\% para Panamá, $13 \%$ para Brasil y el $32 \%$ para Perú. Sin embargo, se sabe que la dominancia de especies raras en captura con redes es evidente, pero poco se conoce sobre la historia natural de estas especies raras, y aún menos sobre como responden a la fragmentación o degradación de sus ambientes (Bierregard \& Stouffer 1997).

Ambos sitios de bosques de coníferas y latifoliadas (Yalmutz y Vivero) presentaron los mayores porcentajes de similitud, compartieron 16 especies, y en donde Mniotilta varia y Melospiza lincolnii fueron exclusivas a estos sitios. El bosque de coníferas y latifoliadas (Vivero) y el bosque de pino-encino (Yalhuech) también presentaron altos porcentajes de similitud, compartiendo también 16 especies, con 4 especies exclusivas (Hylocharis leucotis, Amazilia cyanocephala, Oncostoma cinereigulare y Atlapetes albinuca) (Cuadro 5). 
La temporada de secas que coincide con la temporada migratoria de las aves, presentó tanto un mayor número de individuos como de especies con respecto a la temporada de lluvias. La temporada de secas es la época de escasez de alimento en donde las especies pueden intensificar su búsqueda de alimento e incrementar las probabilidades de ser capturadas. En contraste, la mayoría de las especies de aves en la región Neotropical se reproducen antes de la estación de lluvias posiblemente como respuesta al aumento en la abundancia de artrópodos durante la temporada de lluvias (Poulin et al. 1992). Quizás por esta razón existe una disminución en las tasas de capturas en redes durante ésta temporada del año, aunque también esta disminución esta influida por la ausencia de las especies migratorias Neotropicales.

Los gremios tróficos en una comunidad son tan diversos como las especies. Esto quizás refleja la disponibilidad de comida, así como la estructura de la vegetación que determina la selección de hábitat de las especies (Thiollay 1992). Los principales gremios presentes en los cuatro sitios fueron los insectívoros, frugívoros y nectarívoros. Algunos gremios sobresalieron por el número de especies como de individuos. Por ejemplo, el gremio de los insectívoros fue el más abundante en número de especies que el resto de los demás gremios tróficos, pero los frugívoros arbóreos sobresalieron en número de individuos. Karr et al. (1990), reportaron que el gremio trófico con mayor número de especies en una comunidad de aves bosques tropicales lluviosos es el de los insectívoros.

El bosque de coníferas y latifoliadas (Vivero) presentó el mayor número de especies del gremio insectívoros que el resto de los sitios. Los insectívoros arbóreos recogedores fueron los más abundantes dentro del gremio de los insectívoros y junto con el bosque de pino-encino (Yalhuech) presentaron el mayor número de especies nectarívoras (5). Las especies de colibríes registradas son endémicas a los bosques de montaña (Howell \& Webb 1995). Según Johns (1991), la mayor abundancia de insectívoros de sotobosque se encuentra en ambientes perturbados, ya que estos generalmente reflejan la pérdida de sotobosque y su asociación con insectos, aunque también esta determinado por el tipo de vegetación. Yalmutz, por otra parte, presentó el mayor número de especies frugívoras e insectívoras arbóreas recogedoras. Este sitio de bosque mixto de coníferas y latifoliadas presenta mayor cobertura arbórea y diversidad vegetal.

La diversidad de aves en el Parque Nacional Lagos de Montebello varió entre los sitios estudiados (Las Grutas, Vivero, Yalmutz y Yalhuech), así como entre temporadas (secas y lluvias). Yalmutz fue uno de los sitios con mayor diversidad de aves, mayor número de especies únicas a este sitio, y raras. Es muy probable que las especies exclusivas a estos sitios sean las más vulnerables a sufrir extinciones locales. Aunque en este sitio no se presentaron actividades humanas intensas, no está exento que estas se incrementen en un futuro. Otro sitio importante fueron Las Grutas, el cual es uno de los últimos remanentes de bosque mesófilo de montaña 
dentro del parque. El Parque Nacional Lagos de Montebello presentó una importante diversidad de especies de aves que parece responder a la variación de ambientes presentes. Sin embargo, la persistencia de las poblaciones de aves y su conservación en la región dependerá en gran medida de la permanencia de ambientes no perturbados así como de la calidad de los ambientes secundarios y manejados.

AGRADECIMIENTOS. Agradecemos a L. Martínez, B. Contreras y R. Castellanos por su ayuda durante el trabajo de campo. A D. Díaz Bonifaz por la elaboración del mapa y a Emmanuel Valencia por la elaboración de las figuras. A R. Rodríguez-Estrella, F. González-García y P. Reyes-Castillo por las sugerencias realizadas al manuscrito las cuales lo mejoraron sustancialmente. Al Consejo Nacional de Ciencia y Tecnología, Fondo Mexicano para la Conservación de la Naturaleza y Sistema de Investigación Benito Juárez (SIBEJ) por su apoyo financiero para el proyecto "Diversidad de vertebrados terrestres en un paisaje con diferentes usos del suelo en la región del Parque Nacional Lagos de Montebello, Chiapas".

\section{LITERATURA CITADA}

Bierregard, R.O., \& P. Stouffer.1997. Understory birds and dynamic habitat mosaics in Amazonian rainforests. Pp. 138-155. In: W.F. Laurance \& R.O.Bierregaard (Eds.). Tropical Forest Remmants: Ecology, Management, and Conservation of Fragmented Communities. University of Chicago Press, Chicago, USA.

Brower, J., J. Zar \& C.Von Ende. 1977. Field and Laboratory Methods for General Ecology. 3a. ed. Wm. C. Brown Publishers, USA.

Carlson, M. 1954. Floral elements of the pine-oak-liquidambar forest of Montebello, Chiapas, México. Bulletin of the Torrey Botanical Club, 81: 387-399.

Eisermann, K. \& U. Schulz. 2005. Birds of a high-altitude cloud forest in Alta Verapaz, Guatemala. Revista de Biología Tropical, 53: 577-594.

Escalante-Pliego, P., A.G. Navarro-Sigüenza \& A.T. Peterson. 1998. Un análisis geográfico, ecológico e histórico de la diversidad de aves terrestres de México. Pp. 279-304. En: T. P. Ramamoorthy, R. Bye, A. Lot \& J. Fa (Eds). Diversidad Biológica de México: orígenes y distribución. Instituto de Biología, Universidad Nacional Autónoma de México. DF.

Fahrig, L. \& W.K. Nuttle. 2005. Population ecology in spatially heterogeneous environments. Pp. 95118. En: G. M. Lovett, C. G. Jones, M. G. Turner \&. K. C. Weathers (Eds.). Ecosystem Function in Heterogeneous Landscapes. Springer-Science, New York, NY, USA.

Flores-Villela, O.A. \& P. Geréz. 1994. Biodiversidad y conservación en México: síntesis sobre vertebrados terrestres, vegetación y uso del suelo. UNAM, CONABIO, México.

García, E.1973. Modificaciones al sistema de clasificación climática de Köppen. Instituto de Geografía, Universidad Nacional Autónoma de México, México.

Gómez de Silva, H., F. González-García \& M. P. Castillas-Trejo. 1999. Birds of the upper cloud foresto f El Triunfo, Chiapas, Mexico. Ornitología Neotropical, 10: 1-26.

Gotelli, N.J. \& A.M. Ellison. 2004. A Primer of Ecological Statistics. Sinauer Associates, Inc., Sunderland, MA, USA.

Hanski, I., J. Kouki \& A. Halkka. 1993. Three explanations of the positive relationship between distribution and abundance of species. Pp. 108-116. En: R.E. Ricklefs \& D. Schluter (Eds). Species diversity in Ecological Communities. The University of Chicago Press, USA.

Horváth, A., I.J. March, \& J.H.D. Wolf. 2001. Rodent diversity and land use in Montebello, Chiapas, Mexico. Studies on Neotropical Fauna and Environment, 36: 169-176. 
Rangel-Salazar et al.: Aves de sotobosque del Parque Nacional Lagos de Montebello

Howell, S.N.G. \& S. Webb.1995. A guide to the birds of Mexico and Northern Central America. Oxford University Press, CA, USA.

INEGI. 1984. Carta de Efectos Climáticos Regionales. Mayo-octubre y noviembre-abril. Las Margaritas. 1:250000 (E15-12-D15-3). México, D. F.

Johns, A.D. 1991. Responses of Amazonian rain forest birds to habitat modification. Journal of Tropical Ecology, 7: 417-437.

Karr, J. 1977. Ecological correlates of rarity in a tropical forest bird communities. Auk, 94: 240-247.

Karr, J. 1985. Turnover rates in tropical forest bird communities. National Geographic Society, 1975. Research Report. pp. 421-425.

Karr, J. 1990. Birds of tropical rainforest comparative biogeography and ecology. Pp. 215-228. En: A. Keast (Ed). Biogeography and ecology of forest bird communities. Academic Publishing. The Hague, Netherlands.

Karr, J.R., S.K. Robinson, J.G. Blake, R.O. Bierregaard Jr. 1990. Birds of four neotropical forests. Pp. 237-269. En: A. H. Gentry (Ed). Four Neotropical Rainforests. Yale University Press, New Haven, Conn. USA.

Krebs, C.J. 1989. Ecological Methodology. Harper Coollins Publishers. New York, USA.

Krebs, C.J. 2001. Ecology. The experimental analysis of distribution and abundance. Addison Wesley Longman, Inc. CA.USA.

March, I.J. \& A. Flamenco. 1996. Evaluación rápida de la deforestación en las áreas naturales protegidas de Chiapas (1970-1993). El Colegio de La Frontera Sur, The Nature Conservancy, U.S. AID. San Cristóbal de Las Casas, Chiapas, México.

National Geographic. 1983. Field guide to the birds of North America. National Geographic Society. USA.

Poulin, B., G. Lefebvre \& R. McNeil. 1992. Tropical avian fenology in relation to abundance and explotation of food resources. Ecology, 73: 2295-2309.

Pueyo, S. 2006. Diversity: between neutrality and structure. Oikos, 112:392-405.

Pyle, P., S.N.G. Howell, R.P. Yunick \& D.F. DeSante. 1987. Identification guide to North American Passerines. Slate Creek Press. Bolonias, CA. USA.

Rangel-Salazar J.L., P.L. Enríquez \& J. H. Vega-Rivera.1993. Riqueza de especies de aves de sotobosque en la Selva Lacandona, Chiapas, México. Revista de Biología Tropical, 41: 273-279.

Rangel-Salazar, J.L., P.L. Enríquez \& T. Will. 2005. Diversidad de aves en Chiapas: prioridades de investigación para su conservación. Pp. 265-123. En: M. González-Espinosa, N. Ramírez-Marcial \& L. Ruíz-Montoya (Eds). Diversidad Biológica en Chiapas. El Colegio de La Frontera Sur. COCYTECH. Plaza y Valdés, S. A. de C. V. México.

Rappole, J.H. \& A.R.Tipton.1992. The evolution of avian migration in the Neotropics. Ornitología Neotropical, 3: 45-55.

Ricklefs, R.E. 1977. Environmental Heterogeneity and Plant Species Diversity: A Hypothesis. American Naturalist, 111: 376-381

Rickelfs, R.E. \& D. Schluter. 1993. Species diversity: regional and historical influences. Pp 350-363. En: R.E. Rickelfs \& D. Schluter (Eds). Species diversity in Ecological Communities. Historical and Geographical Perspectives. University of Chicago Press, Chicago USA.

Rzedowski, J. 1978. Vegetación de México. Limusa, México, DF. 432p.

Sall, J., L. Creighton \& A. Lehman. 2005. JMP Start Statistics. SAS Institute Inc. $3^{\text {th }}$ Ed. SAS Institute Inc. Thompson Learning, Belmont, California, USA.

SEMARNAP. 1998. Los incendios forestales en México. Secretaría de Medio Ambiente Recursos Naturales y Pesca. México.

Stiles, G. \& A. Skutch. 1989. A guide to the birds of Costa Rica. Comstock Cornell, USA. 
Terborgh, J. 1985. The vertical component of plant species diversity in temperate and tropical forests. American Naturalist, 126: 760-777.

Terborgh, J., S.K. Robinson, T.A. Parker III, C.A. Munn \& N. Pierpont. 1990. Structure and organization of an Amazonian forest bird community. Ecological Monograph, 60: 213-238.

Thiollay, J.M. 1992. Influence of selective loading on bird species diversity in a Guianan Rain Forest. Conservaion Biology, 6: 47-63.

Winker, K., P. Escalante, J.H. Rappole, M.A. Ramos, R.J. Oehleschlanger \& D.W. Warner. 1997. Periodic migration and lowland forest refuge in a sedentary Neotropical bird, Wetmore's Bush Tanager. Conservation Biology, 11: 692-697.

Zarco, P. 2000. Análisis de la vegetación y fauna asociada en Bosques Templados del Parque Nacional Lagunas de Montebello, Chiapas. Tesis de Licenciatura, Universidad Nacional Autónoma de México, México, D. F. 